\title{
Influence of glycaemic index on subjective appetite responses in healthy adults
}

\author{
M.S. Hafiz ${ }^{1,2}$, M. D. Campbell ${ }^{3,4,5}$, G. Mappa ${ }^{6}$, N. Orsi ${ }^{6}$, C. Orfila ${ }^{1}$ and C. Boesch ${ }^{1}$ \\ ${ }^{1}$ School of Food Science and Nutrition, University of Leeds, Leeds, UK, \\ ${ }^{2}$ Faculty of Applied Medical Sciences, Department of Clinical Nutrition, King Abdul-Aziz University, Jeddah, \\ Saudi Arabia, \\ ${ }^{3}$ Faculty of Health Sciences and Wellbeing, University of Sunderland, UK, \\ ${ }^{4}$ Wellcome-MRC Institute of Metabolic Science, University of Cambridge, UK, \\ ${ }^{5}$ Leeds Institute of Cardiovascular and Metabolic Medicine, University of Leeds, UK and \\ ${ }^{6}$ Leeds Institute of Cancer \& Pathology, St James's University Hospital, Leeds, UK
}

The glycaemic index (GI) of carbohydrate foods is a useful tool for predicting postprandial glucose and insulin responses ${ }^{(1)}$. Results from human studies suggest an inverse relationship between GI of ingested food and postprandial appetite responses ${ }^{(2)}$. However, evidence on the effects of GI on subjective and objective satiety responses is inconclusive ${ }^{(3)}$. Therefore, the aim of this study was to assess the impact of GI on postprandial appetite response in humans.

We conducted a randomised controlled crossover trial on 13 adults presenting with normoglycaemia, normal BMI and mean age of $28.7 \pm 6.6$ years. Participants were randomly allocated to consume a high GI meal (HGI) consisting of mashed potatoes, and low GI (LGI) meal of canned whole chickpeas, each providing $50 \mathrm{~g}$ total available carbohydrates, after an overnight fast. Postprandial subjective satiety and appetite responses were captured using visual analogue scale (VAS) in 3 domains: hunger, fullness, and prospective food intake. Plasma ghrelin values were also measured by immunoassay (intra-assay variations $<15 \%$ ), in venous blood samples collected over 3 hours following breakfast intake. Postprandial total area under the curves (AUCs) were calculated by the trapezoidal method, and data were analysed using t-test with statistical significance set at $p<0.05$. Data are presented as mean \pm SD.

There were great variations observed in individual responses after intake of both interventions in all outcomes illustrated above. Postprandial hunger responses were significantly lower after ingestion of LGI meal $(91.2 \pm 37.1 \mathrm{~mm} \times \mathrm{h})$ in comparison to HGI meal $(113.5 \pm 26 \mathrm{~mm} \times \mathrm{h})(\mathrm{p}=0.035)$; and postprandial fullness responses were significantly higher as demonstrated by AUC values over the 3 hour period following breakfast intake $(107.3 \pm 37.1 \mathrm{~mm} \times \mathrm{h})$ and $(80.2 \pm 24.7 \mathrm{~mm} \times \mathrm{h})(\mathrm{p}=0.012)$ respectively. However, there was no significant difference in prospective food intake following different GI meals $(104.6 \pm 41.9 \mathrm{~mm} \times \mathrm{h}) \mathrm{and}(126.2 \pm 26.3$ $\mathrm{mm} \times \mathrm{h}$ ), respectively. In addition, there were no differences observed in postprandial ghrelin response $(\mathrm{p}>0.05)$.

The results suggest that a low meal GI positively affects postprandial satiety response. However, the impact on appetite hormone ghrelin is unclear possibly due to high individual variances in hormonal responses.

\section{References}

1. Krog-Mikkelsen I, Sloth B, Dimitrov D et al. (2011) J Nutr 141, 1679-1684.

2. Ford H \& Frost G (2010) Proc Nutr Soc 69, 199-203.

3. Niwano Y, Adachi T, Kashimura J et al. (2009) J Nutr Sci Vitaminol 55, 201-207. 